\title{
The second moment of Dirichlet twists of Hecke $L$-functions
}

by

\author{
Peng Gao (Singapore), Rizwanur Khan (Los Angeles, CA) \\ and Guillaume Ricotta (Bordeaux)
}

1. Introduction. Let $f$ be a fixed holomorphic Hecke eigenform of level 1 and weight $k$. For $\Im(z)>0$ we have a Fourier expansion of $f$ :

$$
f(z)=\sum_{n \geq 1} a_{f}(n) n^{(k-1) / 2} e^{2 \pi i n z},
$$

where the coefficients $a_{f}(n)$ are real and satisfy the Ramanujan-Petersson bound $|a(n)| \leq d(n)$, and we have normalized so that $a_{f}(1)=1$. Let $\chi$ be a Dirichlet character of modulus $q$, and form the Dirichlet series of the twist of $f$ by $\chi$ given by

$$
L(f \times \chi, s)=\sum_{n \geq 1} \frac{a_{f}(n) \chi(n)}{n^{s}}=\prod_{p}\left(1-\frac{a_{f}(p) \chi(p)}{p^{s}}+\frac{\chi\left(p^{2}\right)}{p^{2 s}}\right)^{-1}
$$

for $\Re(s)>1$. When $\chi$ is primitive this is an $L$-function, which continues to an entire function and satisfies the functional equation

$$
\begin{aligned}
\left(\frac{q}{2 \pi}\right)^{s} \Gamma(k / 2+s) L( & f \times \chi, 1 / 2+s) \\
& =\iota_{\chi}\left(\frac{q}{2 \pi}\right)^{-s} \Gamma(k / 2-s) L(f \times \bar{\chi}, 1 / 2-s),
\end{aligned}
$$

where $\imath_{\chi}=i^{k} \tau(\chi)^{2} / q$ and $\tau(\chi)$ is the Gauss sum associated to $\chi$ (thus $\left.\left|\imath_{\chi}\right|=1\right)$; cf. [3, Prop. 14.20].

For a large modulus $q$, we are interested in the second power moment of $L(f \times \chi, 1 / 2)$ as $\chi$ ranges over all primitive characters modulo $q$. Stefanicki [7] proved

$$
\sum_{\chi \bmod q}^{*}|L(f \times \chi, 1 / 2)|^{2}=K P_{q}(1) \psi(q) q \log q+O\left(2^{\nu(q)} q(\log q)^{0.935}\right),
$$

2010 Mathematics Subject Classification: Primary 11M99.

Key words and phrases: mean values of $L$-functions. 
where $*$ denotes summation only over primitive characters, $K$ is a constant, $P_{q}(1) \psi(q)$ is a finite Euler product over the prime divisors of $q$, and $\nu(q)$ is the number of distinct prime factors of $q$. A modulus $q$ may have $\frac{\log q}{\log \log q}$ distinct prime factors, but the main term in (1.2) fails to dominate the error term when $\nu(q)$ is larger than $\frac{1}{10} \log \log q$. The condition $\nu(q)<\frac{1}{10} \log \log q$ holds for very few integers $q$, a set of zero density in fact, by a theorem of Hardy and Ramanujan [1]. We are interested in the following problem: can we establish an asymptotic for the second moment for a larger class of integers q; if not all integers, then almost all? This can be considered an analogue of a problem studied by Soundararajan: in [6], he adapts HeathBrown's [2] asymptotic formula for the fourth power moment of Dirichlet $L$-functions to hold for all moduli. Unfortunately, his methods do not seem to immediately carry over to this case and we must do something different. We should also mention that it is an open problem to find an asymptotic expression with a power saving error term for the left hand side of (1.2), even for prime $q$. The corresponding problem for the fourth power moment of Dirichlet $L$-functions with prime moduli was recently solved by Young [8].

Let

$$
K=\frac{6(4 \pi)^{k}}{\pi \Gamma(k)}\|f\|^{2}
$$

where the norm is the Petersson norm. Define the multiplicative function $\psi(q)$ by setting $\psi(p)=(1-2 / p)$ and $\psi\left(p^{k}\right)=(1-1 / p)^{2}$ for $k \geq 2$, so that $q \psi(q)$ is the number of primitive characters modulo $q$. Let

$$
P_{q}(s)=\prod_{p \mid q}\left(1-\frac{1}{p^{s}}\right)^{2}\left(1-\frac{a_{f}(p)^{2}-2}{p^{s}}+\frac{1}{p^{2 s}}\right)\left(1-\frac{1}{p^{2 s}}\right)^{-1} .
$$

Our main theorem is

THEOREM 1.1. For integers q satisfying

$$
\sum_{\substack{p \mid q \\ p>x}} \frac{1}{p} \leq(\log \log q)^{-10}
$$

where

$$
x=\exp \left(\frac{\log \log q}{200 \log \log \log q}\right)
$$

we have

$$
\sum_{\chi \bmod q}^{*}|L(f \times \chi, 1 / 2)|^{2}=K P_{q}(1) \psi(q) q \log q\left(1+O\left((\log \log q)^{-1}\right)\right),
$$

where the implied constant depends on $f$. 
Condition (1.3) holds when for example $\nu(q) \ll \exp \left(\frac{\log \log q}{300 \log \log \log q}\right)$, which is certainly true for almost all integers. We will need the following consequence of this condition.

LEMMA 1.2. If (1.3) holds then

$$
\sum_{\substack{d \mid q \\ d(\log q)^{0.05}}} \frac{|\mu(d)| \prod_{p \mid d}(1+10 / \sqrt{p})}{d} \ll(\log \log q)^{-9} .
$$

Proof. Using "Rankin's trick" we have

$$
\begin{aligned}
\sum_{\substack{d \mid q \\
d \geq(\log q)^{0.05}}} & \frac{|\mu(d)| \prod_{p \mid d}(1+10 / \sqrt{p})}{d} \\
& \leq \sum_{\substack{d|q \\
p| d \Rightarrow p \leq x}}\left(\frac{d}{(\log q)^{0.05}}\right)^{(\log x)^{-1}} \frac{|\mu(d)| \prod_{p \mid d}(1+10 / \sqrt{p})}{d} \\
& \ll(\log \log q)^{-10} \prod_{p \leq x}\left(1+\frac{1}{p^{1-(\log x)^{-1}}}\right) \ll(\log \log q)^{-9} .
\end{aligned}
$$

Thus

$$
\begin{aligned}
& \sum_{\substack{d \mid q \\
d \geq(\log q)^{0.05}}} \frac{|\mu(d)| \prod_{p \mid d}(1+10 / \sqrt{p})}{d} \\
& \ll\left(\sum_{\substack{p \mid q \\
p>x}} \frac{1}{p}\right)\left(\sum_{d \mid q} \frac{|\mu(d)| \prod_{p \mid d}(1+10 / \sqrt{p})}{d}\right)+(\log \log q)^{-9} .
\end{aligned}
$$

This completes the proof as the sum over divisors in the last line of (1.7) is $\ll \prod_{p \mid q}(1+1 / p) \ll \log \log q$.

Throughout the paper all implied constants may depend implicitly on $f$.

2. Proof of Theorem 1.1. The following standard results can be found in Sections 2 and 3.1 respectively of [7].

Lemma 2.1 (Orthogonality). For $(n m, q)=1$ we have

$$
\sum_{\chi \bmod q}^{*} \chi(n) \bar{\chi}(m)=\sum_{\substack{d \mid q \\ n \equiv m \bmod q / d}} \mu(d) \phi(q / d) .
$$


LEMma 2.2 (Approximate functional equation). If $\chi$ is a primitive character modulo $q$ then

$$
|L(f \times \chi, 1 / 2)|^{2}=\sum_{n, m} \frac{a_{f}(n) a_{f}(m) \chi(n) \bar{\chi}(m)}{\sqrt{n m}} V\left(\frac{n m}{q^{2}}\right),
$$

where

$$
V(x)=\frac{1}{\pi i} \int_{(c)} G(y) x^{-y} \frac{d y}{y}
$$

for any $c>0$, and

$$
G(s)=\frac{\Gamma(k / 2+s)^{2}}{(2 \pi)^{2 s} \Gamma(k / 2)^{2}}
$$

decays rapidly in vertical lines. We have $V(x) \ll_{C} \min \left\{1, x^{-C}\right\}$ for any $C>0$, so that the sum above is essentially supported on $n m<q^{2+\varepsilon}$ for any $\varepsilon>0$.

Thus we have

$$
\begin{aligned}
\sum_{\chi \bmod q}^{*} \mid L(f & \times \chi, 1 / 2)\left.\right|^{2} \\
& =\sum_{n, m} \sum_{\chi \bmod q}^{*} \frac{a_{f}(n) a_{f}(m)}{\sqrt{n m}} V\left(\frac{n m}{q^{2}}\right) \sum_{\chi \bmod q}^{*} \chi(n) \bar{\chi}(m) \\
& =\sum_{d \mid q} \mu(d) \phi(q / d) \sum_{\substack{n \equiv m \bmod q / d \\
(m n, q)=1}} \frac{a_{f}(n) a_{f}(m)}{\sqrt{n m}} V\left(\frac{n m}{q^{2}}\right) .
\end{aligned}
$$

The main idea is to treat separately the cases $d<(\log q)^{0.05}$ and $d \geq$ $(\log q)^{0.05}$. Note that in the main term of the theorem, we have $P_{q}(1) \psi(q) \gg$ $(\log \log q)^{-6}$, so we need to bound any error terms by $q \log q(\log \log q)^{-7}$.

\section{Small divisors}

LEMma 2.3. We have, for some $\theta>0$,

$$
\sum_{\substack{n \neq m \\ n=m \bmod l \\(n m, q)=1}} \frac{a_{f}(n) a_{f}(m)}{\sqrt{n m}} V\left(\frac{n m}{q^{2}}\right) \ll \phi(l)^{-1} q(\log q)^{1-0.065}+q^{\varepsilon} l^{-\theta} .
$$

The above lemma is a combination of Lemmas 2, 3 and 7 of [7]. For $n$ and $m$ both close to $l$, a shifted convolution problem is solved to get a power saving bound. For $n$ or $m$ much larger than $l$, the sum is bounded absolutely by appealing to Shiu's [5] estimate for sums of multiplicative functions in arithmetic progression combined with Rankin's [4] result that $\left|a_{f}(n)\right|$ is bounded by $(\log n)^{-0.065}$ on average. This gives only a log power 
saving. The remaining ranges of $n$ and $m$ are trivially bounded with power savings. Thus we have

$$
\begin{aligned}
& \sum_{\substack{d \mid q \\
d<(\log q)^{0.05}}} \mu(d) \phi(q / d) \sum_{\substack{n \equiv m \bmod q / d \\
n \neq m \\
(m n, q)=1}} \frac{a_{f}(n) a_{f}(m)}{\sqrt{n m}} V\left(\frac{n m}{q^{2}}\right) \\
& \ll \sum_{\substack{d \mid q \\
d<(\log q)^{0.05}}}|\mu(d)| \phi\left(\frac{q}{d}\right)\left(\phi\left(\frac{q}{d}\right)^{-1} q(\log q)^{0.935}+q^{\varepsilon}\left(\frac{q}{d}\right)^{-\theta}\right) \\
& \ll q(\log q)^{0.985} .
\end{aligned}
$$

Now we turn to the "diagonal" terms, i.e. those with $n=m$ :

$$
\sum_{\substack{d \mid q \\ d<(\log q)^{0.05}}} \mu(d) \phi\left(\frac{q}{d}\right) \sum_{\substack{n \\(n, q)=1}} \frac{a_{f}(n)^{2}}{n} V\left(\frac{n^{2}}{q^{2}}\right) .
$$

First note that by Lemma 1.2 we have

$$
\begin{aligned}
\sum_{\substack{d \mid q \\
d(\log q)^{0.05}}} \mu(d) \phi\left(\frac{q}{d}\right) & =\sum_{d \mid q} \mu(d) \phi\left(\frac{q}{d}\right)+O\left(q(\log \log q)^{-9}\right) \\
& =q \psi(q)+O\left(q(\log \log q)^{-9}\right) .
\end{aligned}
$$

The last line follows by putting $n=m=1$ in Lemma 2.1. The sum over $n$ can be written as an integral involving the Rankin-Selberg $L$-function $L(f \otimes f, s)$ to get

LEMMA 2.4.

$$
\begin{aligned}
\sum_{\substack{n \\
(n, q)=1}} \frac{a_{f}(n)^{2}}{n} & V\left(\frac{n^{2}}{q^{2}}\right) \\
& =K P_{q}(1) \log q+K_{1} P_{q}(1)+K_{2} P_{q}^{\prime}(1)+O\left(q^{-1 / 5}\right),
\end{aligned}
$$

for some constants $K_{i}$ and

$$
K=\text { residue }_{s=1} 2 L(f \otimes f, s)=\frac{6(4 \pi)^{k}}{\pi \Gamma(k)}\|f\|^{2} .
$$

This is shown in Section 4 of [7], where it is also observed that $P_{q}^{\prime}(1) \ll$ $\nu(q) P_{q}(1)$. Now as $\nu(q) \ll \frac{\log q}{\log \log q}$, we find that the diagonal terms give the main term of Theorem 1.1. 
Large divisors. For $d \mid q$ we can express the condition $n \equiv m \bmod q / d$ using the Dirichlet characters modulo $q / d$. We have

$$
\begin{aligned}
\phi(q / d) & \sum_{\substack{n \equiv m \bmod q / d \\
(m n, q)=1}} \frac{a_{f}(n) a_{f}(m)}{\sqrt{n m}} V\left(\frac{n m}{q^{2}}\right) \\
& =\sum_{\substack{n, m \\
(m n, q)=1}} \sum_{\bmod q / d} \frac{a_{f}(n) a_{f}(m) \chi(n) \bar{\chi}(m)}{\sqrt{n m}} V\left(\frac{n m}{q^{2}}\right) .
\end{aligned}
$$

Now the last line equals

$$
\begin{aligned}
& \quad \frac{1}{\pi i} \int_{(c)} \sum_{\chi \bmod q / d} L(f \times \chi, s+1 / 2) L(f \times \bar{\chi}, s+1 / 2) \\
& \times \prod_{p \mid d}\left(1-\frac{a_{f}(p) \chi(p)}{p^{s+1 / 2}}+\frac{\chi\left(p^{2}\right)}{p^{2 s+1}}\right) \prod_{p \mid d}\left(1-\frac{a_{f}(p) \bar{\chi}(p)}{p^{s+1 / 2}}+\frac{\bar{\chi}\left(p^{2}\right)}{p^{2 s+1}}\right) G(s) q^{2 s} \frac{d s}{s} .
\end{aligned}
$$

Here the extra Euler products arise due to the condition $(n m, q)=1$. On taking $c=1 / \log q$ and using Lemma 3.2 from the final section, we observe that (2.10) is less than a constant multiple of

$$
\frac{q}{d} \log q \log \log q \prod_{p \mid d}\left(1+\frac{10}{\sqrt{p}}\right) \int_{1 / \log q}\left|(s+1)^{4} G(s) q^{2 s} \frac{d s}{s}\right| .
$$

The last integral is easily found to be $\ll \log \log q$. Thus we have

$$
\begin{gathered}
\sum_{\substack{d \mid q \\
(\log q)^{0.05}}} \mu(d) \phi(q / d) \sum_{\substack{n \equiv m \bmod q / d \\
(m n, q)=1}} \frac{a_{f}(n) a_{f}(m)}{\sqrt{n m}} V\left(\frac{n m}{q^{2}}\right) \\
\ll q \log q(\log \log q)^{2} \sum_{\substack{d \mid q \\
d \geq(\log q)^{0.05}}} \frac{|\mu(d)| \prod_{p \mid d}(1+10 / \sqrt{p})}{d} .
\end{gathered}
$$

This is $\ll q \log q(\log \log q)^{-7}$ by Lemma 1.2 .

3. An upper bound for the second moment. In this section we will need a different form of the approximate functional equation.

Lemma 3.1 (Approximate functional equation). For a primitive character $\chi$ modulo $q$ and $\Re(s)=0$ we have 


$$
\begin{aligned}
L(f \times \chi, 1 / 2+s)= & \sum_{n} \frac{a_{f}(n) \chi(n)}{n^{1 / 2+s}} W_{s}\left(\frac{n}{q}\right) \\
& +\iota_{\chi} \frac{(2 \pi)^{2 s} \Gamma(k / 2-s)}{q^{2 s} \Gamma(k / 2+s)} \sum_{n} \frac{a_{f}(n) \bar{\chi}(n)}{n^{1 / 2-s}} W_{-s}\left(\frac{n}{q}\right),
\end{aligned}
$$

where

$$
W_{s}(x)=\frac{1}{2 \pi i} \int_{(c)} \frac{\Gamma(k / 2+s+y)}{\Gamma(k / 2+s)} e^{y^{2}}(2 \pi x)^{-y} \frac{d y}{y}
$$

for any $c>0$. We have $W_{s}(x) \ll_{C} \min \left\{1,|s+1|^{C} x^{-C}\right\}$ for any $C>0$, so that the sums above are essentially supported on $n<q^{1+\varepsilon}$ for any $\varepsilon>0$.

This is a special case of Theorem 5.3 in [3]. The bound on $W_{s}(x)$ can be seen by moving the line of integration far to the right when $x>1$ and just to the left of the origin when $x \leq 1$.

We show the following.

LEMma 3.2. For $\Re(s) \geq 0$ and integers $q$ and $r$ we have

$$
\begin{aligned}
& \mid \sum_{\chi \bmod q} L(f \times \chi, 1 / 2+s) L(f \times \bar{\chi}, 1 / 2+s) \\
& \times \prod_{p \mid r}\left(1-\frac{a_{f}(p) \chi(p)}{p^{s+1 / 2}}+\frac{\chi\left(p^{2}\right)}{p^{2 s+1}}\right)\left(1-\frac{a_{f}(p) \bar{\chi}(p)}{p^{s+1 / 2}}+\frac{\bar{\chi}\left(p^{2}\right)}{p^{2 s+1}}\right) \mid \\
& \ll|s+1|^{4} q \log q \log \log q \prod_{p \mid r}\left(1+\frac{10}{\sqrt{p}}\right) .
\end{aligned}
$$

Proof. The statement of the lemma certainly holds for $\Re(s) \geq 2$, so by the Phragmén-Lindelöf principle (cf. [3, Theorem 5.53]) it is enough to prove the result for $\Re(s)=0$. Thus throughout this proof, assume that $s$ is purely imaginary. First we demonstrate the bound

$$
\sum_{\chi \bmod d}^{*}|L(f \times \chi, 1 / 2+s)|^{2} \ll|s+1|^{4} d \log d .
$$

By Lemma 3.1 it is enough to bound

$$
\begin{aligned}
& \sum_{\chi \bmod d}^{*}\left|\sum_{n} \frac{a_{f}(n) \chi(n)}{n^{1 / 2+s}} W_{s}\left(\frac{n}{d}\right)\right|^{2} \\
& \ll \sum_{\chi \bmod d}\left|\sum_{n \leq d} \frac{a_{f}(n) \chi(n)}{n^{1 / 2+s}} W_{s}\left(\frac{n}{d}\right)\right|^{2}+\sum_{\chi \bmod d}\left|\sum_{n>d} \frac{a_{f}(n) \chi(n)}{n^{1 / 2+s}} W_{s}\left(\frac{n}{d}\right)\right|^{2} .
\end{aligned}
$$

By a large sieve inequality (cf. [3, Section 7.5]) and the Rankin-Selberg 
bound $\sum_{n \leq x} a_{f}(n)^{2} \ll x$, we have

$$
\sum_{\chi \bmod d}\left|\sum_{n \leq d} \frac{a_{f}(n) \chi(n)}{n^{1 / 2+s}} W_{s}\left(\frac{n}{d}\right)\right|^{2} \ll d \sum_{n \leq d} \frac{a_{f}(n)^{2}}{n} \ll d \log d .
$$

The remaining sum in (3.4) equals, by orthogonality,

$$
\phi(d) \sum_{\substack{1 \leq r \leq d \\(r, d)=1}}\left|\sum_{\substack{n>d \\ n \equiv r \bmod d}} \frac{a_{f}(n)}{n^{1 / 2+s}} W_{s}\left(\frac{n}{d}\right)\right|^{2} .
$$

If we drop the condition $(r, d)=1$, this is bounded by

$$
\begin{aligned}
& d \sum_{h \geq 0} \sum_{n>d} \frac{a_{f}(n) a_{f}(n+h d)}{n^{1 / 2+s}(n+h d)^{1 / 2-s}} W_{s}\left(\frac{n}{d}\right) \overline{W_{s}\left(\frac{n+h d}{d}\right)} \\
& \ll d \sum_{h \geq 0} \sum_{n>d} \frac{a_{f}(n)^{2}+a_{f}(n+h d)^{2}}{n^{1 / 2} d^{1 / 2}}\left(\frac{d}{n}\right)(1+h)^{-3}|1+s|^{4} \ll d|1+s|^{4} .
\end{aligned}
$$

This establishes (3.3).

Now, the left hand side of (3.2) is bounded by

$$
\prod_{p \mid r}\left(1+\frac{10}{\sqrt{p}}\right) \sum_{\chi \bmod q}|L(f \times \chi, 1 / 2+s)|^{2} .
$$

We have

$$
\begin{aligned}
\sum_{\chi \bmod q} \mid L(f & \times \chi, 1 / 2+s)\left.\right|^{2}=\left|L\left(f \times \chi_{0}, 1 / 2+s\right)\right|^{2} \\
& +\sum_{d \mid q} \sum_{\chi \bmod d}^{*}|L(f \times \chi, 1 / 2+s)|^{2} \prod_{p \mid q / d}\left|1-\frac{a_{f}(p) \chi(p)}{p^{1 / 2+s}}+\frac{\chi\left(p^{2}\right)}{p^{2 s}}\right|^{2} .
\end{aligned}
$$

Using (3.3) we have

$$
\begin{aligned}
\sum_{\chi \bmod q}|L(f \times \chi, 1 / 2+s)|^{2} & \ll|s+1|^{4} \sum_{d \mid q} d \log d \prod_{p \mid q / d}\left(1+\frac{10}{\sqrt{p}}\right) \\
& \ll|s+1|^{4} q \log q \prod_{p \mid q}\left(1+\frac{1}{p}\right) \\
& \ll|s+1|^{4} q \log q \log \log q .
\end{aligned}
$$

Acknowledgements. We would like to thank Prof. K. Soundararajan for his encouragement. We also thank the referee for a careful reading and helpful suggestions on exposition. The first author is supported by a research fellowship from an Academic Research Fund Tier 1 grant at Nanyang Technological University. The second author is partially supported by the Na- 
tional Science Foundation. The third author is financed by the ANR project "Aspects Arithmétiques des Matrices Aléatoires et du Chaos Quantique".

\section{References}

[1] G. H. Hardy and S. Ramanujan, The normal number of prime factors of a number n, [Quart. J. Math. 48 (1917), 76-92], in: Collected Papers of Srinivasa Ramanujan, AMS Chelsea Publ., Providence, RI, 2000, 262-275.

[2] D. R. Heath-Brown, The fourth power mean of Dirichlet's L-functions, Analysis 1 (1981), 25-32.

[3] H. Iwaniec and E. Kowalski, Analytic Number Theory, Amer. Math. Soc. Colloq. Publ. 53, Amer. Math. Soc., Providence, RI, 2004.

[4] R. A. Rankin, Sums of powers of cusp form coefficients. II, Math. Ann. 272 (1985), 593-600.

[5] P. Shiu, A Brun-Titchmarsh theorem for multiplicative functions, J. Reine Angew. Math. 313 (1980), 161-170.

[6] K. Soundararajan, The fourth moment of Dirichlet L-functions, in: Analytic Number Theory, Clay Math. Proc. 7, Amer. Math. Soc., Providence, RI, 2007, 239-246.

[7] T. Stefanicki, Non-vanishing of L-functions attached to automorphic representations of $\mathrm{GL}(2)$ over $\mathbb{Q}$, J. Reine Angew. Math. 474 (1996), 1-24.

[8] M. Young, The fourth moment of Dirichlet L-functions, http://arxiv.org/abs/ math.NT/0610335.

Division of Mathematical Sciences

Nanyang Technological University

21 Nanyang Link

Singapore 637371

E-mail: penggao@ntu.edu.sg

Institut de Mathématiques de Bordeaux

Université de Bordeaux 1

Laboratoire A2X, Bâtiment A33, Bureau 301 M

351 cours de la Libération

33405 Talence Cedex, France

E-mail: guillaume.ricotta@math.u-bordeaux1.fr
Department of Mathematics University of California, Los Angeles 520 Portola Plaza Los Angeles, CA 90095-1555, U.S.A. E-mail: rrkhan@math.ucla.edu

Received on 13.12.2008

and in revised form on 4.3.2009 